\title{
Expressional Modulation of Aquaporin 1 and 9 in the Rat Epididymis by an Anabolic-Androgenic Steroid, Nandrolone Decanoate
}

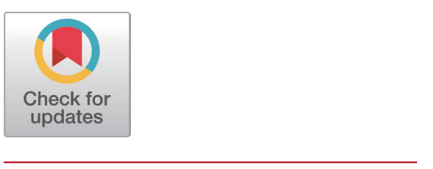

Received: September 6, 2021

Revised: October 30, 2021

Accepted: November 19, 2021

${ }^{+}$Corresponding author

Ki-Ho Lee

Dept. of Biochemistry and Molecular

Biology, Eulji University, Daejeon 34824,

Korea.

Tel: +82-42-259-1643

Fax: +82-42-259-1649

E-mail: kiholee@eulji.ac.kr

Copyright ๑ 2021 The Korean Society of Developmental Biology.

This is an Open Access article distributed under the terms of the Creative Commons Attribution Non-Commercial License (http://creativecommons.org/licenses/ by-nc/4.0/) which permits unrestricted non-commercial use, distribution, and reproduction in any medium, provided the original work is properly cited.

ORCID

$\mathrm{Ki}$-Ho Lee

https://orcid.org/0000-0002-3495-5126

Conflict of interests

The author declares no potential conflict of interest.

Acknowledgements

This research was supported by Basic Science Research Program through the National Research Foundation of Korea (NRF) funded by the Ministry of Education (2017R1D1A1B03030209).

\section{Authors' contributions}

The article is prepared by a single author.

\section{Ethics approval}

All procedures were carried out in accordance with the guidelines for the care and use of laboratory animals of National Research Council in Korea.
${ }^{\dagger} \mathrm{Ki}$-Ho Lee

Dept. of Biochemistry and Molecular Biology, College of Medicine, Eulji University, Daejeon 34824, Korea

\section{Abstract}

The spermatozoa become mature in the epididymis which is divided into initial segment and caput, corpus, and cauda epididymis. The water movement across the epididymal epithelium is important for creating luminal microenvironment for sperm maturation. Aquaporins (Aqps) are water channel proteins, and expression of Aqps is regulated by androgens. The current research was focused to examine expressional regulation of Aqp1 and Aqp9 by an androgenic-anabolic steroid, nandrolone decanoate (ND). The ND at the low dose (2 mg/ $\mathrm{kg}$ body weight/week) or high dose (10 mg) was subcutaneously administrated into male rats for 2 or 12 weeks. Transcript levels of Aqp1 and Aqp9 were determined by quantitative real-time polymerase chain reaction (PCR) analyses. In the initial segment, level of Aqp1 was decreased with 12 week-treatment, while Aqp9 level was decreased by the high dose treatment for 12 weeks. In the caput epididymis, Aqp 9 expression was decreased by the low dose treatment. The 2 week-treatment resulted in an increase of Aqp1 level but a decrease of Aqp9 expression in the corpus epididymis. In the corpus epididymis, the 12 week-treatment at the low dose caused the reduction of Aqp1 and Aqp9 levels, but the high dose treatment resulted in an increase of Aqp1 expression and a decrease of Aqp9 level. In the cauda epididymis, Aqp1 expression was decreased by 2 and 12 week-treatments, while increases of Aqp9 levels was detected with the high dose treatment for 2 weeks and with 12 week-treatment. These findings indicate differential regulation of Aqp1 and Aqp9 expression among epididymal segments by ND.

Keywords: Epididymis, Aquaporin 1, Aquaporin 9, Real-time polymerase chain reaction (PCR), Nandrolone decanoate

\section{INTRODUCTION}

The male reproductive tract is consisted of the testis, excurrent ductules, and accessory glands, including prostate and seminal vesicle. The epididymis is a part of excurrent ductules, which the testis is connected via efferent ductules (Robaire et al., 2006). The epididymis has a tubular structure, which the lumen is located inside and is surrounded by a layer of epithelial cells (Robaire et al., 2006). The epididymis is anatomically divided into four parts, including initial segment, caput (head 
region) epididymis, corpus (body region) epididymis, and cauda (tail region) epididymis, which are characterized by the presence and different frequency of specific cell types and different histochemical existence of enzymatic molecules (Robaire et al., 2006). The epididymal cell types present within the epithelial layer are principal, apical, narrow, clear, basal, and halo cells (Robaire et al., 2006). Even though the principal cell is found along the entire epididymal ductules, the restricted localization of other cell types is commonly observed (Robaire et al., 2006). For example, the apical and narrow cells are mainly found in the initial segment (Sun \& Flickinger, 1980), while the clear cells only exist in the caput, corpus, and cauda epididymis (Abou-Haila \& Fain-Maurel, 1984). In addition, a number of researches have demonstrated that these cell types have distinctive morphologies, functions, and localization relative to other cell types (Robaire et al., 2006). Thus, it is generally considered that each epididymal region plays different roles on control and regulation of epididymal functions.

The epididymis is the site in which immature spermatozoa produced from the testis become mature and acquire motility and fertilizing capacity (Robaire et al., 2006). The physiological functions of epididymis include transport and maturation of spermatozoa and storage and protection of spermatozoa (Robaire et al., 2006). The regulation of water movement into and out of the epididymal lumen is a way to form the adequate luminal microenvironment for sperm maturation (Robaire et al., 2006). Even though most of testicular fluid is reabsorbed in the efferent ductules (Clulow et al., 1994), luminal water reabsorption at significant level occurs in the epididymis, especially in the caput epididymis (Tao et al., 2000). Other scientific evidences have also shown that not only fluid reabsorption from the epididymal lumen but also water secretion into the lumen are equally important to regulate the luminal composition of the epididymis and the fluidity of the epididymal luminal content (Wong, 1998). Thus, the controlled transepithelial water movement is important to regulate sperm maturation of epididymal function.

Aquaporins (AQP) are water channel molecules that are chiefly localized at plasma membrane in various cell types, including testis, efferent ductules, and epididymis in the male reproductive tract (Verkman \& Mitra, 2000; Da Silva et al., 2006). AQPs have 6 membrane-spanning domains in structure and contribute to water movement across plasma membrane (Verkman \& Mitra, 2000; Ribeiro et al., 2021). The AQPs have at least 13 isoforms in mammalian cells, and expression and localization of different types of AQPs in the male reproductive tract have been extensively examined (Da Silva et al., 2006; Ribeiro et al., 2021). AQPs are also capable of transporting ammonia or hydrogen peroxide, depending on types of AQPs (Da Silva et al., 2006; Ribeiro et al., 2021). There are at least 8 types of AQPs expressing in the epididymis, and specific expression of different $\mathrm{AQP}$ types has been detected along the epididymis (Ribeiro et al., 2021). For examples, expression of AQP1 is found in smooth muscle and endothelial cells adjacent to epididymal epithelial cells (Brown et al., 1993), and AQP9 is exclusively localized at the apical microvilli of principal cells throughout the entire epididymal region (Pastor-Soler et al., 2001). The expression of AQPs in the male reproductive tract is regulated by various factors, including androgen and estrogen (Badran \& Hermo, 2002; PastorSoler et al., 2002; Oliveira et al., 2005). Because expressional alterations of AQPs in male reproductive tract are associated with the reduction of reproductive capability (Huang et al., 2006), it is relatively reasonable to consider that exogenous compound affecting male fertility could influence expression of $\mathrm{AQPs}$ in the male reproductive tract.

Nandrolone decanoate (ND) is an androgenic-anabolic steroidal compound which is frequently administrated to treat several clinical symptoms, such as muscle wasting accompanied with human immunodeficiency virus infection and anemia involved in chronic renal failure (Busardò et al., 2015). Uncontrolled and unregulated usage of ND is strongly associated with the reduction of male fertility, probably due to expressional alteration of steroidogenic enzymes in the testis in part (Min 
\& Lee, 2018). In addition, chronic administration of ND results in a decrease of epididymal weight (Mirkhani et al., 2005), even though the effect of ND on the epididymal has not determined in detail. Non-clinical use of ND among non-professional people and bodybuilders appears to be increased (de Souza \& Hallak, 2011). But, despite deleterious undesired outcomes on male fertility by the misuse of ND, the effect of ND on the epididymis has been rarely studied.

The present research was focused to determine the effect of ND treatment at different doses for short or long period on expression of $A q p 1$ and $A q p 9$ in the rat epididymis. The segmental expressional modulation of $A q p 1$ and $A q p 9$ by ND treatment was examined by using a quantitative real-time polymerase chain reaction $(\mathrm{PCR})$ analysis.

\section{MATERIALS AND METHODS}

\section{Experimental animals, nandrolone decanoate treatment, and tissue collection}

A total of 34 male Sprague Dawley rats at 40 days of age were purchased from Samtako (Osan, Korea) and individually caged during an entire experimental period. The animals were allowed ad libitum access to food and water and kept in controlled conditions. The ND, $50 \mathrm{mg} / \mathrm{mL}$ concentration in a stock solution, was obtained from Organon Korea (Seoul, Korea), and diluted into adequate concentrations with peanut oil for ND treatment.

The equal number of animals at 50 days of age were divided into two different treatment groups, 2 week-ND treatment and 12 week-ND treatment groups. The animals in each experimental group were further randomly assigned into one of three subgroups, including control (peanut oil treatment, $\mathrm{n}=5$ ), low dose-ND treatment ( $2 \mathrm{mg}$ of $\mathrm{ND} / \mathrm{kg}$ body weight/week, $\mathrm{n}=6$ ), or high dose$\mathrm{ND}$ treatment $(10 \mathrm{mg}$ of ND/kg body weight/week, $\mathrm{n}=6$ ). The first subcutaneous ND injection was started at 50 days of age, and the injection amount of peanut oil or ND was adjusted every week by measuring body weight of animal.

After the last injection was allowed at the day of 2 nd or 12 th week, the animal was euthanized by over-exposure to $\mathrm{CO}_{2}$ in a closed chamber. The reproductive tract, including testis and epididymis, was pulled out through an incision made on the scrotum. The epididymis was rapidly separated from the testis and efferent ductules in cold phosphate-buffered saline (PBS) solution. The epididymis transferred into a fresh PBS solution was further dissected out into initial segment, caput epididymis, corpus epididymis, and caudal epididymis. The epididymal segments were quickly frozen in liquid nitrogen and stored in $-80^{\circ} \mathrm{C}$ until further use.

\section{Total RNA isolation and quantitative real-time polymerase chain reaction (PCR)}

Total RNA from the frozen tissue was extracted with Trizol reagent (Molecular Research Center, Cincinnati, OH, USA). Shortly, the tissue was rapidly homogenized in Trizol solution, and chloroform and isopropanol were sequentially supplied to precipitate total RNA. The concentration of total RNA re-suspended in DEPC- $\mathrm{dH}_{2} \mathrm{O}$ was measured by NanoDrop Lite spectrophotometer (Thermo Scientific, Massachusetts, MA, USA). The quality of total RNA was determined by $1.2 \%$ agarose gel electrophoresis.

One microgram of total RNA was used to generate the first strand complementary DNA (cDNA) strand by utilizing iScrip ${ }^{\mathrm{TM}}$ Reverse transcription Supermix for reverse transcription (RT)-qPCR (Bio-Rad Laboratories, Hercules, CA, USA). A total volume of the mixture with total RNA was adjusted with nuclease-free $\mathrm{dH}_{2} \mathrm{O}$ to $20 \mu \mathrm{L}$. The RT reaction was carried out at $25^{\circ} \mathrm{C}$ for $5 \mathrm{~min}, 46^{\circ} \mathrm{C}$ for $20 \mathrm{~min}$, and $95^{\circ} \mathrm{C}$ for $1 \mathrm{~min}$ as instructed in the protocol.

The quantitative real-time PCR was performed with a mixture of $1 \mu \mathrm{L}$ of $\mathrm{cDNA}, 7 \mu \mathrm{L}$ of $\mathrm{iQ}^{\mathrm{TM}}$ 
SYBR $^{\circledR}$ Green Supermix (Bio-Rad Laboratories), 10 pmol of each oligonucleotide primer, and nuclease-free $\mathrm{dH}_{2} \mathrm{O}$ to make a total volume of $25 \mu \mathrm{L}$. The information of oligonucleotide primer used for real-time PCR is shown in Table 1. As an internal control for PCR analysis, cyclophilin A (Ppia) was used. The PCR was executed in a pre-denaturation step at $95^{\circ} \mathrm{C}$ for $5 \mathrm{~min}$, cycles of a denaturation step at $95^{\circ} \mathrm{C}$ for $30 \mathrm{sec}$, an annealing step at $\mathrm{T}_{\mathrm{m}}$ for $30 \mathrm{sec}$, and an extension step at $72^{\circ} \mathrm{C}$ for $30 \mathrm{sec}$ in PTC-200 Chromo 4 real-time system (Bio-Rad Laboratories). At the end of each PCR, an extra extension step at $72{ }^{\circ} \mathrm{C}$ for 10 min was added. The PCR product was fractured in $1.2 \%$ agarose gel electrophoresis to check the size.

\section{Statistical analysis and data presentation}

The levels of $A q p 1$ and $A q p 9$ transcripts in each experimental groups were adjusted to that of Ppia transcript level. The relative ratios of $A q p 1$ and $A q p 9$ expression levels among experimental groups were obtained from $2^{-\Delta \Delta C t}$ method (Livak \& Schmittgen, 2001). Independently triplicated or quadruplicated RT reactions and PCRs were performed to acquire a mean and standard error of an experimental groups, and the data were presented in the relative ratio of $A q p 1$ or $A q p 9$ transcript level against that of control group. The one-way ANOVA was employed to determined the existence of statistical differences at transcript level of $A q p 1$ or $A q p 9$ among different experimental groups. If a significance was detected, a post-hoc test, Duncan's test, was followed. Less than 0.05 level of probability was considered to be statistically different.

\section{RESULTS}

\section{Expressional changes of Aqp1 and Aqp9 in the rat epididymal initial segment by nan- drolone decanoate (ND) treatment for 2 weeks or 12 weeks}

The transcript level of $A q p 1$ in the initial segment was not significantly changed by ND treatment for 2 weeks (Fig. 1A). The similar finding was observed from Aqp9 transcript level treated with ND for 2 weeks (Fig. 1B). However, a significant decrease of $A q p 1$ expression level was detected in the initial segment by ND treatment at the low dose for 12 weeks (Fig. 1C). The level of Aqp1 transcript at the high dose-treated group of ND for 12 weeks was also significantly lower than that of control group, even though there was no statistical difference between the low and high dose-treated groups of ND (Fig. 1C). The level of $A q p 9$ transcript was not significantly changed by ND treatment at the low dose for 12 weeks, while the high dose treatment of ND for 12 weeks resulted in a decrease of $A q p 9$ expression in the initial segment (Fig. 1D).

\section{Expressional changes of Aqp 1 and Aqp9 in the rat caput epididymis by nandrolone decanoate (ND) treatment for 2 weeks or 12 weeks}

The expression of $A q p 1$ in the caput epididymis with ND treatment for 2 week was not

Table 1. Primer information for real-time PCR analysis

\begin{tabular}{llcc}
\hline \hline \multicolumn{1}{c}{ Gene } & \multicolumn{1}{c}{ Sequence $\left(5^{\prime} \rightarrow 3^{\prime}\right)$} & $\mathrm{T}_{\mathrm{m}}\left({ }^{\circ} \mathrm{C}\right)$ & PCR product (bps) \\
\hline $\begin{array}{l}\text { Aqp1 } \\
\text { (NM_012778.2) }\end{array}$ & (F) GTCCCACATGGTCTAGCCTT & 65 & 364 \\
$\begin{array}{l}\text { Aqp9 } \\
\text { (NM_022960.2) }\end{array}$ & $\begin{array}{l}\text { (F) GAAGCTCGACCCAGACATGA } \\
\text { (R) GTAAAAAAGGTCTCTGAGGCTATCCA }\end{array}$ & 65 & 374 \\
$\begin{array}{l}\text { Ppia } \\
\text { (NM_017101) }\end{array}$ & $\begin{array}{l}\text { (F) GGCAAATGCTGGACCAAACAC } \\
\text { (R) TTAGAGTTGTCCACAGTCGGAGATG }\end{array}$ & 62 & \multirow{2}{*}{196} \\
\hline
\end{tabular}

PCR, polymerase chain reaction; Aqp1, aquaporin 1; Aqp9, aquaporin 9; and Ppia: cyclophilin A. 
(A)

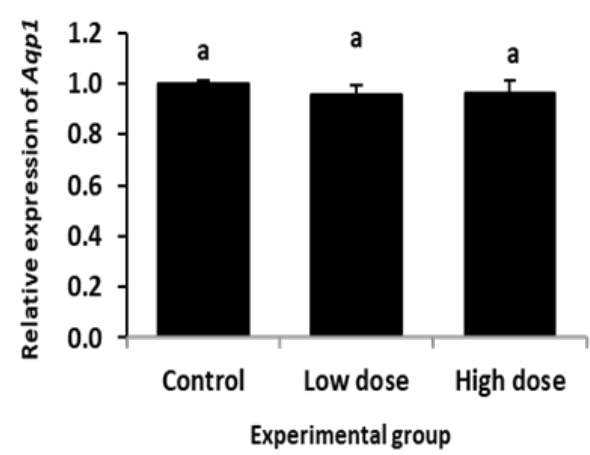

(C)

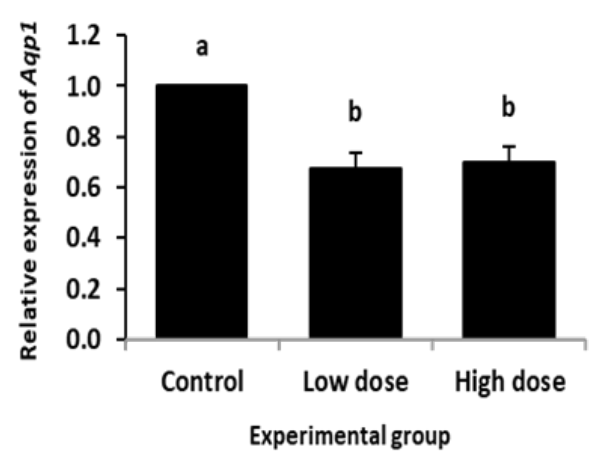

(B)

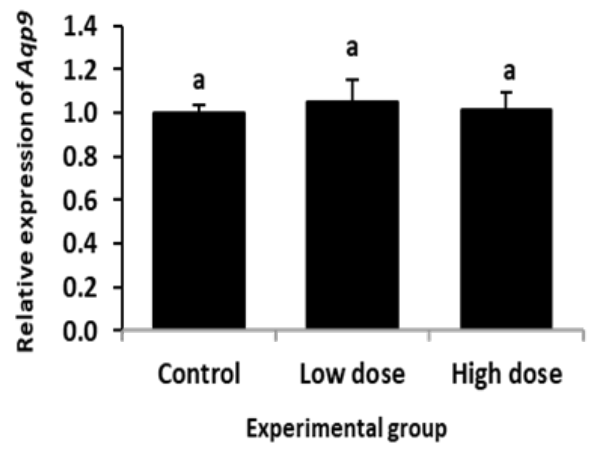

(D)

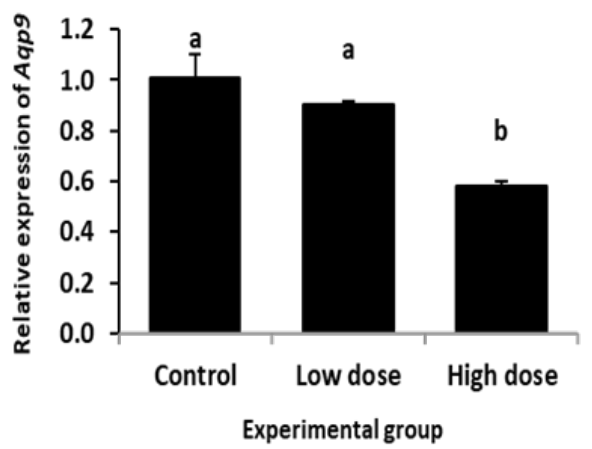

Fig. 1. Expression changes of Aqp1 and Aqp9 in the initial segment of rat epididymis after ND treatment for 2 weeks or 12 weeks at different doses. The animals were treated with ND for 2 weeks (A and B) or 12 weeks (C and D) at 0 (control), 2 (low dose), or 10 (high dose) $\mathrm{mg} / \mathrm{kg}$ body weight/week. The levels of Aqp1 ( $A$ and $C$ ) and Aqp9 (B and D) transcripts were normalized to that of Ppia. ${ }^{a, b}$ Different letters on bars indicate statistical differences among experimental groups at $p<0.05$. Aqp1, aquaporin 1; Aqp9, aquaporin 9; Ppia, cyclophilin A; ND, nandrolone decanoate.

significantly changed, regardless the dose of treatment (Fig. 2A). However, a significant decrease of Aqp 9 transcript level was detected in the caput epididymis with ND treatment at the low dose for 2 weeks, while there was no significant change of $A q p 9$ transcript level at the high dose ND treatedgroup for 2 weeks (Fig. 2B).

The expression patterns of $A q p 1$ and $A q p 9$ in the caput epididymis by ND treatment for 12 weeks (Fig. 2C and D) were quietly similar with those of ND treatment for 2 weeks (Fig. 2A and B). That is, the ND treatment for 2 weeks didn't give an influence on the level of Aqp1 transcript at any dose (Fig. 2C). But, the low dose treatment of ND for 12 weeks induced a reduction of $A q p 9$ expression in the caput epididymis, even though there was no significant expression change of $A q p 9$ observed with the high dose ND treatment for 12 weeks (Fig. 2D).

\section{Expressional changes of Aqp1 and Aqp9 in the rat corpus epididymis by nandrolone decanoate (ND) treatment for 2 weeks or 12 weeks}

Fig. 3 shows the changes of $A q p 1$ and $A q p 9$ transcript levels in the rat corpus epididymis treated with ND for 2 or 12 weeks. The ND treatment at the low dose for 2 weeks resulted in a significant increase of $A q p 1$ transcript level in the corpus epididymis (Fig. 3A). A further increase of $A q p 1$ 
(A)

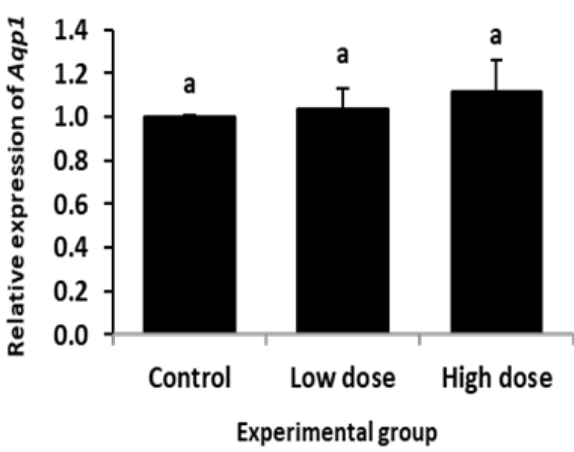

(C)

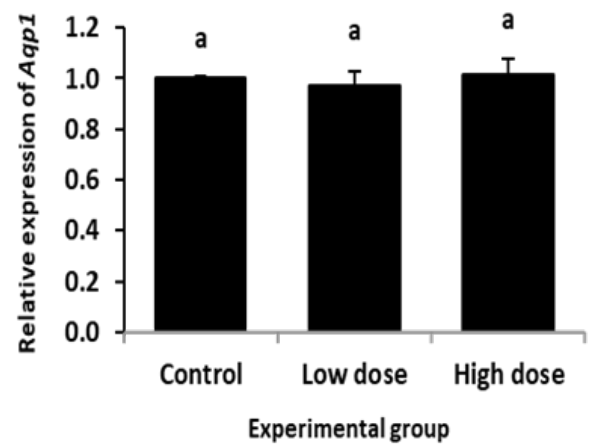

(B)

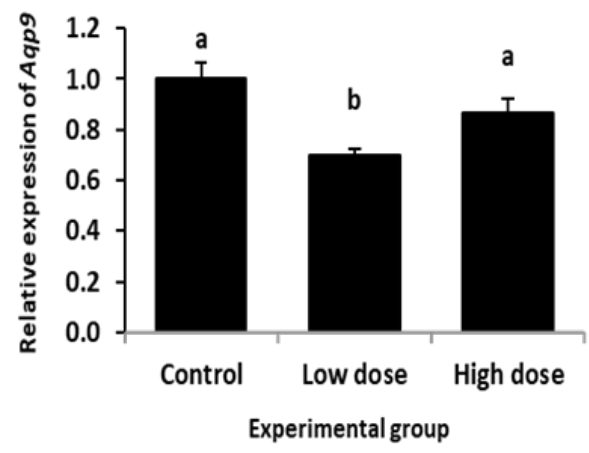

(D)

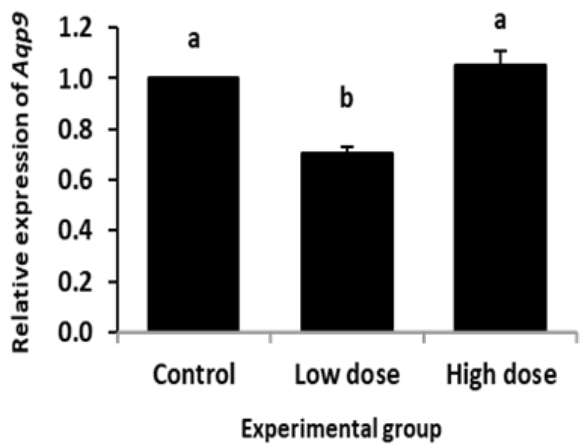

Fig. 2. Expression changes of Aqp1 and Aqp9 in the rat caput epididymis after ND treatment for 2 weeks or 12 weeks at different doses. The animals were treated with ND for 2 weeks ( $A$ and $B$ ) or 12 weeks (C and $\mathrm{D}$ ) at 0 (control), 2 (low dose), or 10 (high dose) mg/kg body weight/week. The levels of Aqp1 ( $\mathrm{A}$ and C) and Aqp9 (B and D) transcripts were normalized to that of Ppia. ${ }^{a, b}$ Different letters on bars indicate statistical differences among experimental groups at $p<0.05$. Aqp1, aquaporin 1; Aqp9, aquaporin 9; Ppia, cyclophilin A; ND, nandrolone decanoate.

transcript amount was observed in the corpus epididymis exposed to the high dose of ND for 2 weeks (Fig. 3A). However, expression of $A q p 9$ was significantly decreased by ND treatment at the low dose for 2 weeks (Fig. 3B). The ND treatment at the high dose for 2 weeks caused an additional reduction of $A q p 9$ transcript level in the corpus epididymis (Fig. 3B).

The level of $A q p 1$ in the corpus epididymis was significantly decreased by the low dose treatment of ND for 12 weeks (Fig. 3C). But, the $A q p 1$ transcript level in the corpus epididymis of the high dose ND treatment groups for 12 weeks was statistically higher than those of control and low dose ND treatment groups (Fig. 3C). Expression of $A q p 9$ in the corpus epididymis by ND treatment for 12 weeks was significantly decreased at both doses (Fig. 3D).

\section{Expressional changes of Aqp 1 and Aqp9 in the rat cauda epididymis by nandrolone decanoate (ND) treatment for 2 weeks or 12 weeks}

In the cauda epididymis, ND treatment for 2 weeks resulted in significant decreases of Aqp1 transcript levels at both doses (Fig. 4A). However, ND treatment at the low dose for 2 weeks didn't influence on the level of $A q p 9$ transcript, even though the high dose treatment of ND for 2 weeks caused a significant increase of $A q p 9$ expression in the cauda epididymis (Fig. 4B). 
(A)

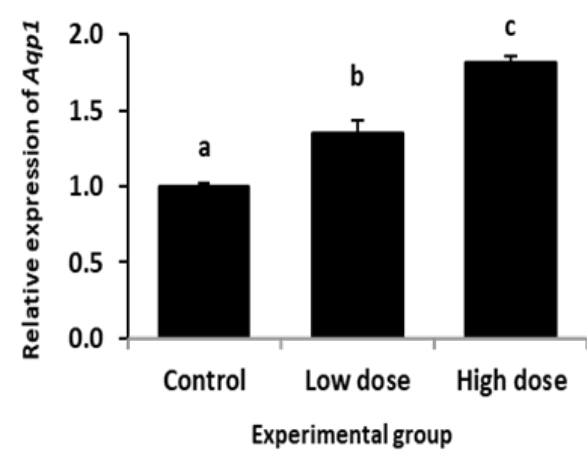

(C)

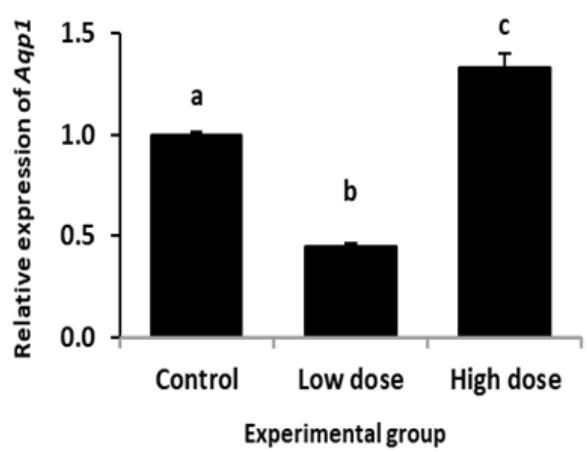

(B)

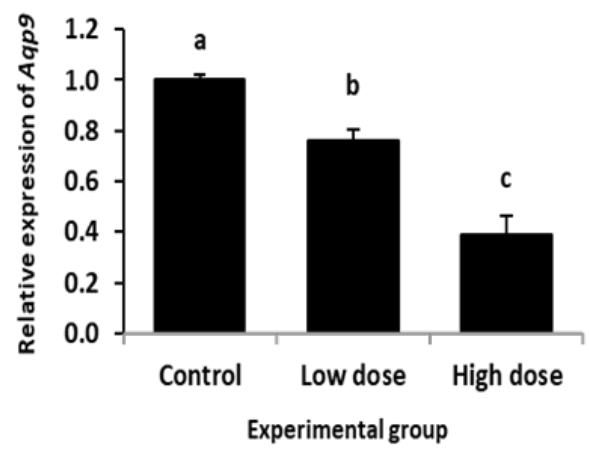

(D)

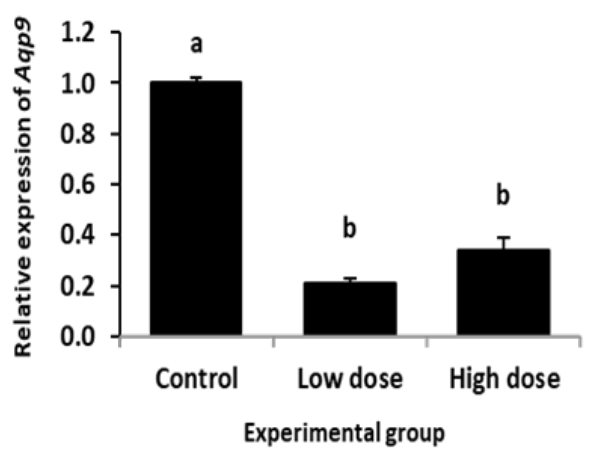

Fig. 3. Expression changes of Aqp1 and Aqp9 in the rat corpus epididymis after ND treatment for 2 weeks or 12 weeks at different doses. The animals were treated with ND for 2 weeks (A and B) or 12 weeks (C and $D$ ) at 0 (control), 2 (low dose), or 10 (high dose) $\mathrm{mg} / \mathrm{kg}$ body weight/week. The levels of Aqp1 ( $\mathrm{A}$ and $\mathrm{C}$ ) and Aqp9 (B and D) transcripts were normalized to that of Ppia. ${ }^{\mathrm{a}-\mathrm{c}}$ Different letters on bars indicate statistical differences among experimental groups at $p<0.05$. Aqp1, aquaporin 1; Aqp9, aquaporin 9; Ppia, cyclophilin A; ND, nandrolone decanoate.

Expression of $A q p 1$ in the cauda epididymis was significantly decreased by the low dose treatment of ND for 12 weeks (Fig. 4C). The level of $A q p 1$ transcript in the caudal epididymis treated with the high dose of ND for 12 weeks was significantly lower than that of control group, but higher than that of the low dose ND treatment group (Fig. 4C). The low dose ND treatment for 12 weeks resulted in a significant increase of $A q p 9$ transcript level in the cauda epididymis (Fig. 4D). An additional increase of $A q p 9$ expression in the cauda epididymis was observed with the high dose ND treatment for 12 weeks (Fig. 4D).

\section{DISCUSSION}

The present research examined expression changes of $A q p 1$ and $A q p 9$ in each epididymal segment by ND treatment at two doses for 2 or 12 weeks. The current findings are summarized as follows: 1) expression of $A q p 1$ and $A q p 9$ is differentially modulated among different epididymal segments by ND treatment, even at same dose for same period; 2) ND treatments at different doses usually result in similar outcomes on expression of $A q p 1$ or $A q p 9$ within a given epididymal 
(A)

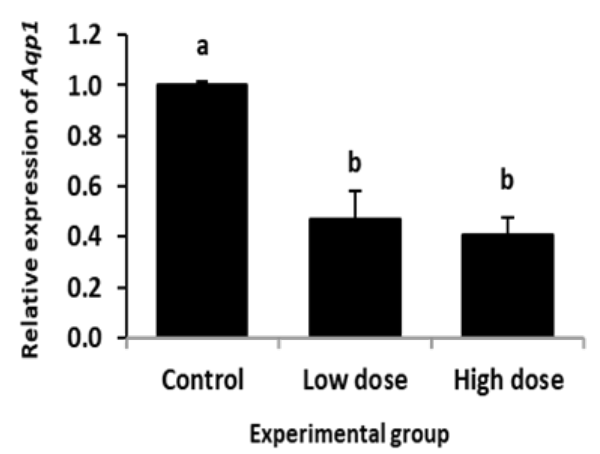

(C)

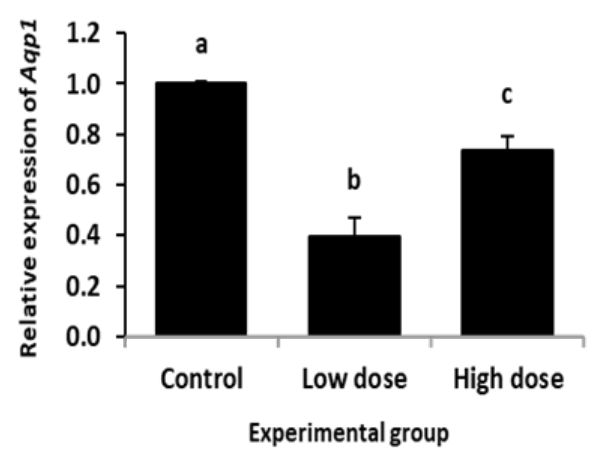

(B)

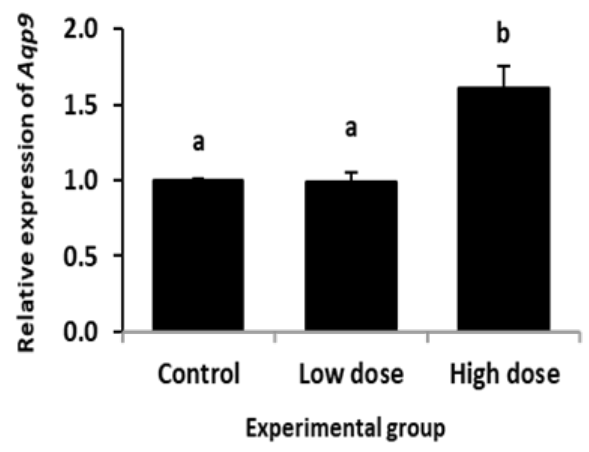

(D)

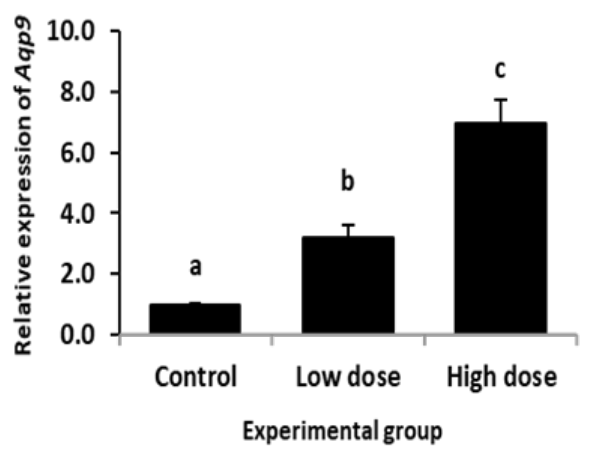

Fig. 4. Expression changes of Aqp1 and Aqp 9 in the rat cauda epididymis after ND treatment for 2 weeks or 12 weeks at different doses. The animals were treated with ND for 2 weeks ( $A$ and $B$ ) or 12 weeks (C and $\mathrm{D}$ ) at 0 (control), 2 (low dose), or 10 (high dose) $\mathrm{mg} / \mathrm{kg}$ body weight/week. The levels of Aqp1 (A and C) and Aqp9 (B and D) transcripts were normalized to that of Ppia. ${ }^{a-c}$ Different letters on bars indicate statistical differences among experimental groups at $p<0.05$. Aqp1, aquaporin 1; Aqp9, aquaporin 9; Ppia, cyclophilin A; ND, nandrolone decanoate.

segment; and 3) change patterns of $A q p 1$ and $A q p 9$ expression by ND treatment in an epididymal segment are not always same, even at same doses and for same period.

Hormonal regulation of $A q p$ s expression in the excurrent duct of male reproductive tract has been demonstrated from other researches. Expression of $A q p 9$ is modulated by estrogen and $5 \alpha$-dihydrotestosterone in the efferent ductules, but only by $5 \alpha$-dihydrotestosterone in the initial segment (Oliveira et al., 2005). The nonsteroidal antiandrogen flutamide results in a decrease of Aqp9 expression in the epididymis (Pastor-Soler et al., 2002). The exposure to a steroidal estrogen receptor antagonist, ICI182,780, leads to a decrease of $A q p 1$ expression in the monkey caput epididymis (Shayu et al., 2005). In addition, 3-beta-diol, an androgenic metabolite, could influence on the regulation of Aqp9 expression in the efferent ductules (Picciarelli-Lima et al., 2006). Moreover, Badran \& Hermo (2002) have shown that expression of $A q p 9$ in the initial segment and cauda epididymis appears to be regulated by testicular factor(s), not by androgen, suggesting differential regulation of specific $A q p$ s at different epididymal segments. Thus, these observations indicate that expressional regulation of $A q p$ s in the efferent ductules and epididymis is under control of various testicular and/or nontesticular factors. Also, expression of specific Aqp type in the epididymis could be regulated by segment-specific manner(s). Indeed, the present research 
have also shown differential expression patterns of $A q p 1$ and $A q p 9$ in different epididymal regions by same dose and/or duration treatment of ND. For example, the ND treatment for 2 weeks has not affected expression of $A q p 1$ and $A q p 9$ in the initial segment, while an increased level of $A q p 1$ transcript and a decreased level of $A q p 9$ transcript have been observed with same treatment in the corpus epididymis. Also, in the caput epididymis, even though the low dose treatments of ND for 2 and 12 weeks result in decreased levels of $A q p 9$ transcript, the high-dose ND treatments for same periods have not influenced on $A q p 9$ expression level. Together with the other's findings, the current research data suggest that expression of $A q p 1$ and $A q p 9$ along the epididymis by the exposure to $\mathrm{ND}$ is differentially regulated in segment-specific and/or dose and treatment duration-dependent manners.

Such differential regulation of $A q p 1$ and $A q p 9$ by ND could be due to different distribution of androgen receptor (AR) concentration along the epididymis. Pujol \& Bayard (1979) have shown that the caput epididymis has the highest $\mathrm{AR}$ concentration and the corpus epididymis has the lowest $\mathrm{AR}$ amount. Because ND has a similar affinity to AR comparable with testosterone (Bergink et al., 1985), ND could exert its biological effect via AR in a target tissue. Thus, it was expected that the caput epididymis would be more responsive than the corpus epididymis to ND exposure. However, more dramatic changes of $A q p 1$ and $A q p 9$ expression by ND treatment have been observed in the corpus epididymis, not in the caput epididymis. In addition, significant changes of transcript levels in the caput epididymis have been just detected in $A q p 9$, not $A q p 1$, exposed to the low dose of ND. A clear answer to such ambiguous data could not be provided at this moment. An additional investigation of the regulatory effect of ND on $A q p 1$ and $A q p 9$ expression in the epididymis is suggested.

It is also suggested that expressional change of $A q p 1$ and $A q p 9$ by ND treatment in the epididymis could be not direct action of ND but indirect effect accompanied with systemic response to ND. The exposure to ND frequently accomplishes aberrant expression of steroidogenic enzymes and abnormal histology in the testis (Svechnikov et al., 2010; Min \& Lee, 2018). In fact, a previous research has demonstrated that ND administration in a same experimental condition with the present study results in significant reduction of several testicular steroidogenic enzymes, including steroidogenic acute regulatory protein, cytochrome $\mathrm{P} 450$ side chain cleavage, and cytochrome $\mathrm{P} 450$ $17 \alpha$-hydroxylase, accompanied with a drastic decrease of serum testosterone concentration (Min \& Lee, 2018). Because functions of the epididymis are largely influenced by testosterone (Robaire et al., 2006), a decrease of testicular testosterone synthesis by ND exposure could give an impact on gene expression in the epididymis to result in functional abnormality. However, even though this suggestion seems to be supported by dose-related decreases of $A q p 9$ transcript levels in the corpus epididymis by 2 week-ND treatment, other data don't seem to fit to the presumption.

The present research shows that expressional regulation of $A q p 1$ and $A q p 9$ in the epididymis by ND is quite complicate. The existence of complex regulatory mechanism of $A q p 1$ and $A q p 9$ in the epididymis by hormonal factor has also been proposed from others (Badran \& Hermo, 2002; Oliveira et al., 2005; Squillacioti et al., 2021). Considering the main function of the epididymis and physiological role of AQPs in the epididymis, it is suggested that the aberrant expression of $A q p 1$ and $A q p 9$ in the epididymis by ND treatment would cause inappropriate development of mature spermatozoa, thus leading into ND-induced infertility. In addition, a possible segment-specific regulation of $A q p 1$ and $A q p 9$ expression in the epididymis by ND administration is suggested from the current study, even though detailed molecular mechanism(s) would be determined from further researches. 


\section{REFERENCES}

Abou-Haila A, Fain-Maurel MA (1984) Regional differences of the proximal part of mouse epididymis: Morphological and histochemical characterization. Anat Rec 209:197-208.

Badran HH, Hermo LS (2002) Expression and regulation of aquaporins 1, 8, and 9 in the testis, efferent ducts, and epididymis of adult rats and during postnatal development. J Androl 23:358-373.

Bergink EW, Janssen PS, Turpijn EW, van der Vies J (1985) Comparison of the receptor-binding properties of nandrolone and testosterone under in vitro and in vivo conditions. J Steroid Biochem 22:831-836.

Brown D, Verbavatz JM, Valenti G, Lui B, Sabolić I (1993) Localization of the CHIP28 water channel in reabsorptive segments of the rat male reproductive tract. Eur J Cell Biol 61:264-273.

Busardò FP, Frati P, Sanzo MD, Napoletano S, Pinchi E, Zaami S, Fineschi V (2015) The impact of nandrolone decanoate on the central nervous system. Curr Neuropharmacol 13:122-131.

Clulow J, Jones RC, Hansen LA (1994) Micropuncture and cannulation studies of fluid composition and transport in the ductuli efferentes testis of the rat: Comparisons with the homologous metanephric proximal tubule. Exp Physiol 79:915-928.

Da Silva N, Piétrement C, Brown D, Breton S (2006) Segmental and cellular expression of aquaporins in the male excurrent duct. Biochim Biophys Acta 1758:1025-1033.

de Souza GL, Hallak J (2011) Anabolic steroids and male infertility: A comprehensive review. BJU Int 108:1860-1865.

Huang HF, He RH, Sun CC, Zhang Y, Meng QX, Ma YY (2006) Function of aquaporins in female and male reproductive systems. Hum Reprod Update 12:785-795.

Livak KJ, Schmittgen TD (2001) Analysis of relative gene expression data using real-time quantitative PCR and the $2^{-\Delta \Delta C_{\mathrm{T}}}$ method. Methods 25:402-408.

Min T, Lee KH (2018) Effects of nandrolone decanoate on expression of steroidogenic enzymes in the rat testis. Asian-Australas J Anim Sci 31:658-671.

Mirkhani H, Golbahar J, Shokri S (2005) Chronic administration of nandrolone decanoate does not increase the plasma homocysteine level of male rats. Basic Clin Pharmacol Toxicol 97:214217.

Oliveira CA, Carnes K, França LR, Hermo L, Hess RA (2005) Aquaporin-1 and -9 are differentially regulated by oestrogen in the efferent ductule epithelium and initial segment of the epididymis. Biol Cell 97:385-395.

Pastor-Soler N, Bagnis C, Sabolic I, Tyszkowski R, McKee M, Van Hoek A, Breton S, Brown D (2001) Aquaporin 9 expression along the male reproductive tract. Biol Reprod 65:384-393.

Pastor-Soler N, Isnard-Bagnis C, Herak-Kramberger C, Sabolic I, Van Hoek A, Brown D, Breton S (2002) Expression of aquaporin 9 in the adult rat epididymal epithelium is modulated by androgens. Biol Reprod 66:1716-1722.

Picciarelli-Lima P, Oliveira AG, Reis AM, Kalapothakis E, Mahecha GAB, Hess RA, Oliveira CA (2006) Effects of 3-beta-diol, an androgen metabolite with intrinsic estrogen-like effects, in modulating the aquaporin-9 expression in the rat efferent ductules. Reprod Biol Endocrinol 4:51.

Pujol A, Bayard F (1979) Androgen receptors in the rat epididymis and their hormonal control. J Reprod Fertil 56:217-222.

Ribeiro JC, Alves MG, Yeste M, Cho YS, Calamita G, Oliveira PF (2021) Aquaporins and (in) fertility: More than just water transport. Biochim Biophys Acta Mol Basis Dis 1867:166039.

Robaire B, Hinton BT (2006) The epididymis. In: Neill JD (ed), Knobil and Neill's Physiology of 
Reproduction. 3rd ed. Elsevier, Amsterdam, Netherlands, pp 1071-1148.

Shayu D, ChennaKesava CS, Soundarajan R, Rao AJ (2005) Effects of ICI 182780 on estrogen receptor expression, fluid absorption and sperm motility in the epididymis of the bonnet monkey. Reprod Biol Endocrinol 3:10.

Squillacioti C, Mirabella N, Liguori G, Germano G, Pelagalli A (2021) Aquaporins are differentially regulated in canine cryptorchid efferent ductules and epididymis. Animals 11:1539.

Sun EL, Flickinger CJ (1980) Morphological characteristics of cells with apical nuclei in the initial segment of the adult rat epididymis. Anat Rec 196:285-293.

Svechnikov K, Izzo G, Landreh L, Weisser J, Söder O (2010) Endocrine disruptors and Leydig cell function.J Biomed Biotechnol 2010:684504.

Tao L, Zupp JL, Setchell BP (2000) Effect of efferent duct ligation on the function of the bloodtestis barrier in rats.J Reprod Fertil 120:13-18.

Verkman AS, Mitra AK (2000) Structure and function of aquaporin water channels. Am J Physiol Renal Physiol 278:F13-F28.

Wong PY (1998) CFTR gene and male fertility. Mol Hum Reprod 4:107-110. 
